\title{
MoleCool: Augmented Reality in Chemistry Education, Possibilities and Solutions
}

\author{
Eszter Lokodi \\ Technical University of Cluj-Napoca, Computer \\ Science Department \\ Cluj-Napoca, Romania \\ lokodi.ga.eszter@utcluj.didatec.ro
}

\author{
Teodor Ștefănut \\ Technical University of Cluj-Napoca, Computer \\ Science Department \\ Cluj-Napoca, Romania \\ teodor.stefanutecs.utcluj.ro
}

\begin{abstract}
Chemistry is a very complex and difficult field to understand in the natural sciences. We all know the popular expression: everything around us is chemistry. Any object we see or touch is chemistry, the food we eat is chemistry, the air we breathe is chemistry, the emotions we experience are chemistry, our body is chemistry. But little do we know or understand how this actually manifests in reality. The current research presents an original solution for chemistry learning, based on Augmented Reality and Gamification principles. The main purpose of this approach is to help students visualize the microscopic molecule structures and describe them in an interactive manner. After a short overview of Augmented Reality initiatives in education the focus switches to the chemistry related initiatives. Finally, the details of the proposal are being discussed.
\end{abstract}

\section{Author Keywords}

Augmented Reality; Chemistry education; Mobile applications.

\section{ACM Classification Keywords}

Interaction paradigms; Human computer interaction (HCI); Human-centered computing; Mixed / Augmented reality;

\section{General Terms \\ Human Factors; Design;}

DOI: 10.37789/rochi.2021.1.1.25

\section{INTRODUCTION}

Educational content can be experienced through a wide variety of environments. Students have traditionally learned through interaction with teachers and colleagues and through non-interactive media, such as textbooks and instructional videos. In the last half century, digital media has entered the educational scene more and more, offering students learning opportunities in interactive simulations and educational games. Serious games are games that have educational purpose besides entertainment. The reasons why game-based learning is so popular are: they improve student involvement and motivation making it easier to memorize and retain knowledge, and students can learn without feeling like they are studying.
Practical activities allow students to make (macroscopic) observations. At the same time, they must be able to explain the observations using ideas about atoms and molecules (sub-microscopic) and chemical symbols (symbolic). It is important to focus on these three levels of representation of chemistry (macroscopic, submicroscopic, and symbolic), which are known as the Johnstone triplet [1] and on how they relate to each other.

\section{EDUCATION AND IMMERSIVE REALITY}

Augmented reality and virtual reality have one important thing in common: both have the remarkable ability to change the user's perception of the world. Where they differ is the perception of the user's presence in these realities. This difference can be illustrated with the help of the concept of Reality-Virtuality Continuum (Figure 1) defined by Paul Milgram and Fumio Kishino [2]. The real world and a total virtual environment are at the two ends of this continuum with the middle region being called Mixed Reality. Augmented reality is close to the end of the realworld spectrum, the predominant perception being the real world improved by the artificially generated data. Instead, augmented virtuality is a term coined by Milgram to identify systems that are mostly synthetic, referring more to Virtual Reality.

Immersive learning is the learning process performed using a simulated or artificial environment. This environment allows students to fully immerse themselves in learning and in a way that they can feel like they are experiencing a proper learning environment. Immersive technologies refer to technologies that change reality and space in which an individual interacts [3]. Fully immersive technology takes the form of virtual reality, while partially immersive technology takes the form of augmented reality.

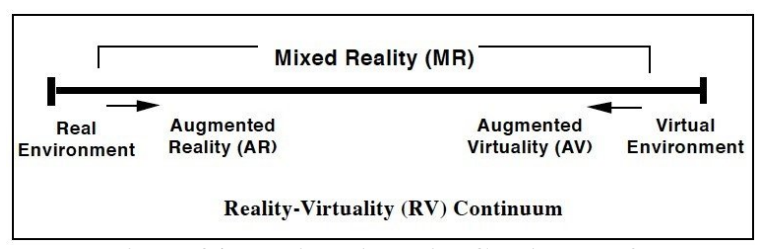

Figure 34. Reality-Virtuality Continuum.[2]

Children born in this digital era are exposed to constant stimuli which contributes to their short attention span. One 
of the many advantages of augmented reality is its capability to enrich the real world and make it reality more captivating [4]. Other advantages of AR include: the possibility of learning through experiencing, individualized learning contributing to inclusivity and increased motivation. Studies by Dalgarno \& Lee [5] and Cheng and Tsai [6] show that AR assisted learning have high impact on student's spatial ability, practical skills and conceptual understanding. Chen in another article [7] affirms that this technology benefits the understanding of chemical structures.

Taking into account the mentioned factors and the past examples, an application on the Android operating system using augmented reality technology was built. For this choice the following advantages of AR technology over virtual reality were considered:

- Lower costs, in the case of AR technology you can use the BYOD method (bring your own device) and the necessary material can be downloaded directly to your own devices;

- Integrates virtual elements in the real world, so the user does not completely separate the information from reality but includes it;

- $\mathrm{AR}$ is more helpful in developing spatial vision capabilities;

- $\quad$ ARCore, the SDK used for Android development is the most advantageous and is considered the most used for AR application development [8].

\section{RELATED WORK}

Applications based on virtual and augmented realities can be found currently in many areas of education, including mathematics (Construct3D [9]), medicine (Medchem VR [10]), preschool education (The Magic Book [11]) and several subdomains of chemistry (organic chemistry [12], stereochemistry [13], inorganic chemistry [14], environmental chemistry [15], etc.). These technologies have already begun to show promise in helping students learn more effectively and increase knowledge retention, compared to traditional 2D desktop interfaces.

EduChem VR [13] is a platform designed as a low-cost system that supports the development of applications with the vision of educating and inspiring by creating RV content easily accessible through smartphone devices, thus reaching anyone anywhere. The company, in order to attract students and inspire further studies, introduces play elements in chemical education through easy-to-use smartphone applications for Google Cardboards and
GearVR. The ability to use the Google Cardboard device to interact with the virtual world makes applications more available because of lower costs.

The basic structure of the atom can be described most simply as a nucleus containing protons and neutrons, surrounded by electrons. Atom Visualizer for ARCore [16] allows the user to place floating patterns of any atoms of the periodic table in the room, then observe them from different angles simply by moving. The Bohr model, which presents the atom as a shell containing a nucleus and electrons in orbit around it, is implicit, but one can also use the more precise quantum mechanical model, which presents the atom as a cloud of electrons. Using the application, the two types of atomic models can be projected in a real-world environment, and the speed of the animation can be adjusted. The nature of the Atom Visualizer application is largely educational and can be used for a more informal science lesson. When viewed in the planetary model, one can understand the energy levels of electrons and their movements around the nucleus, while viewing the quantum mechanic model of an atom helps to understand the possible location of electrons relative to the nucleus.

The Augmented Chemistry and Augmented Chemical Reactions applications focus on combining the visualization of complex structures and the advantages of direct manipulation of physical objects. Both systems use enhanced reality for visualization and a marker tracker to provide direct manipulation of the 3D user interface to control the position and orientation of virtual objects.

Augmented Chemistry (AC) [17] uses a tangible user interface (TUI) for organic chemistry education. It is implemented as a workbench consisting of a table and a projection screen. Just below the screen is a camera that records user interaction and this image in the "magic" mirror is then enriched with virtual elements. Users interact with the models from this virtual environment using a brochure, a cube, a platform and the so-called Gripper. The augmented 3D models are represented through acual markers which can be manipulated by the user in order to select, position, rotate, compose 3D models, thus interacting with the virtual environment.

Augmented Chemical Reactions (ACR) [18] is a 3D visualization tool for presenting and controlling molecule models in an intuitive way, as well as the dynamics of atoms in and between molecules. The purpose of the application is to provide to the students an intuitive tool for learning chemistry and a scientific tool for examining design problems in the initial stage of designing new 


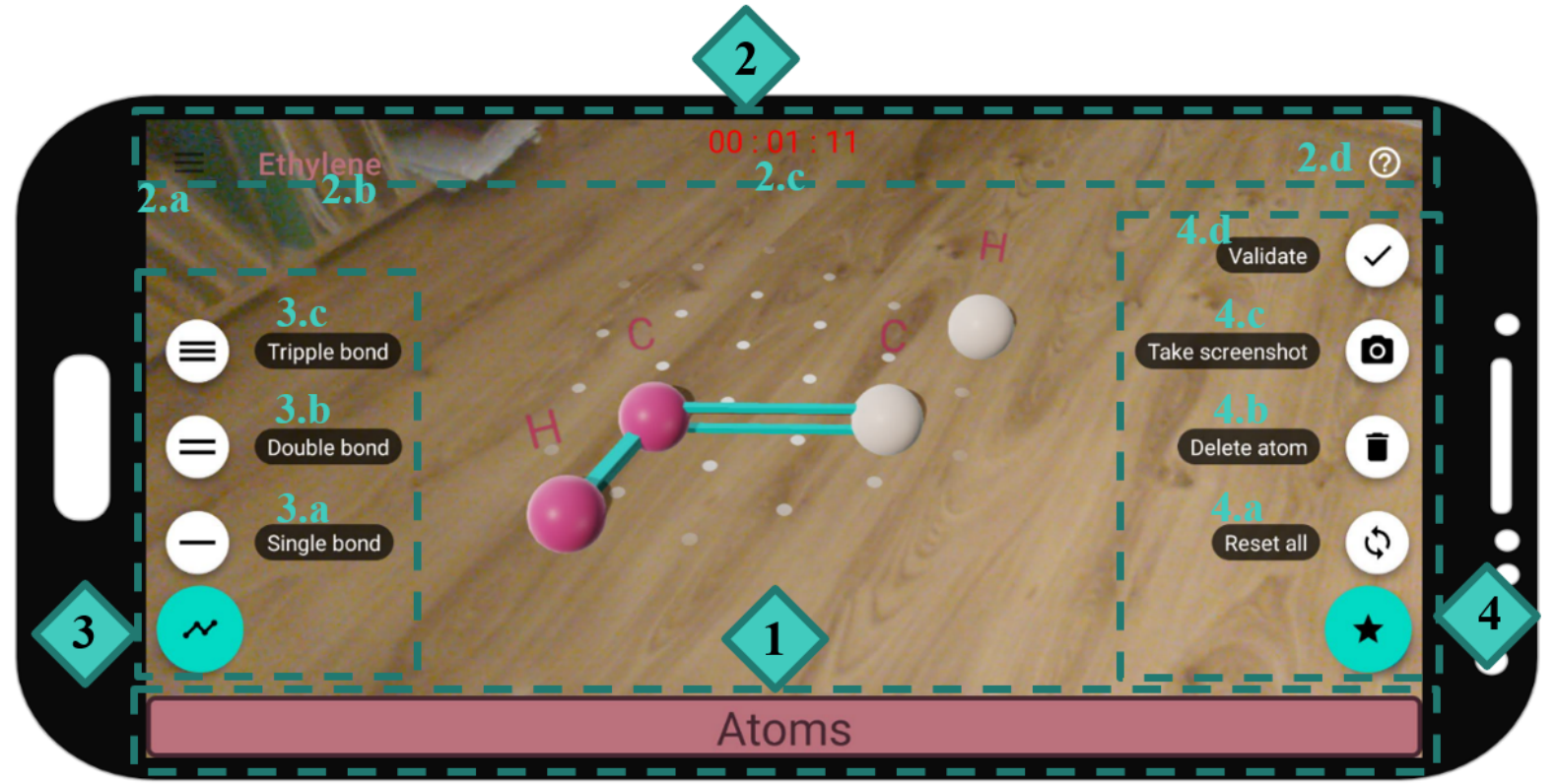

Figure 35. Possible user interactions while interacting with the AR Scene.

molecules. The system incorporates the Augmented Reality (AR) paradigm, where users can manipulate virtual $3 \mathrm{D}$ objects in a simple and intuitive way by moving real cube-like objects with visual markers on the sides. A realtime simulation calculates the forces of attraction and repulsion between the reactants of a molecule as they are moved in the interaction space. The movement of tangible substituents for reactants leads to a dynamic deformation in the structure of the possible product of the molecule.

The approaches mentioned above are mainly focused on different visualization techniques of Chemistry elements in AR environments. Next to visualization, this solution also encourages creativity and gamification inspired activities, allowing the user to recreate in the virtual space known molecules while checking correctness and measuring performance. All these features are meant to stimulate memorization of chemical structures through repetition and visual memory. Just like in the other applications, the virtual elements can be rendered and viewed from different angles, but what is new is that the user can also directly interact with these elements.

\section{PROPOSED SOLUTION}

Based on the research a mobile application was impelented, currently available only for the Android operating system using augmented reality. The application aims to practice molecular structures in $3 \mathrm{D}$ and abstract complex concepts such as atoms, bonds between them and molecules.

\section{Implementation details}

The application has a client-server architecture. The server side consist of a PostgreSQL relational database and a Spring application which communicates with it. An external database for storing the chemical structures in order to be able to add new molecules without reinstalling the application was choosen. The client side consists of an Android application developed in Java.

\section{Augmented Reality Component}

The charm of the application is integrated AR technology. For integrating augmented reality the chosen technologies were ARCore [19] and Sceneform SDK [20]. Based on Perkins Coie and the XR Association's Augmented and Virtual Virtual Reality Survey conducted in 2020 [8] Google ARCore is considered the most used development platform in AR applications. ARCore itself is not an SDK, rather an engine that helps SDKs display objects. Therefore, to use this functionality, Google has released the Sceneform SDK to allow developers to build Android AR applications without having to learn OpenGL or 3D graphics.

For user interactions with the virtual 3D objects, ARCore uses hit testing on the screen of the device by considering a coordinate ( $\mathrm{x}, \mathrm{y}$ ) (given by a touch, tap or other cooperation supported by the application) and a ray in the camera perspective of this current reality. Through these elements it can identify any planes or feature points on which the radius intersects, along with the posture of that intersection in space. This allows users to choose or generally interact with objects in the environment. 
For interacting with augmented reality objects several classes were used from ARCore and Sceneform like: ArFragment, Plane, Scene, HitTestResult, Node, Anchor, AnchorNode, Vector3, Quaternion, ViewRenderable, ModelRenderable, ShapeFactory, etc.

\section{User Interaction}

When opening the application the user is greeted by the splash screen containing the application's logo. After a few seconds he/she is redirected to the Home page, where a guide on how to use the application is displayed. With the help of a navigation drawer placed on the upper-left corner the user can navigate between the different destinations: Home, Play game, Scores.

The Play game destination represents the main section of the application. On open it displays a list of molecular structures that have been previously defined in the database. The information is not stored locally on the device but is retrieved through an online call to the application server. This architectural aspect provides the necessary support for the future implementation of real-life teaching scenarios, where teachers can define a set of molecular structures to be rehearsed by the students.

The game itself starts on the AR screen (Figure 2), after a molecule is selected from the list. Every time a game is started there is a help dialog with the instructions displayed on how to interact with the user interface. The application turns on the camera of the mobile device and lets the user see the surrounding reality and interact with it through the screen. Detection of planes begins instantly. When a plane is found it is marked by covering with a dotted pattern. All the indicated planes are recognized and ready to be used by the user for placing 3D virtual elements.

In this case the user renders 3D models for different atoms. These models are made out of a sphere and the atom's symbol above it. The type of the atom can be defined from the Bottom Sheet (Figure 3). The augmented interface is also equipped with graphical interface elements to define different functionalities. These can be divided into four groups:

1. Bottom Sheet: This menu can be accessed by pressing or dragging the bar at the bottom with the name Atoms. It contains a list from which the user can choose the type of atom to be placed on the plane. Once chosen, by tapping the screen at a point where a plane has been identified, a sphere symbolizing the atom is projected in the virtual space. For clarity, the short name of the atom also appears above it.

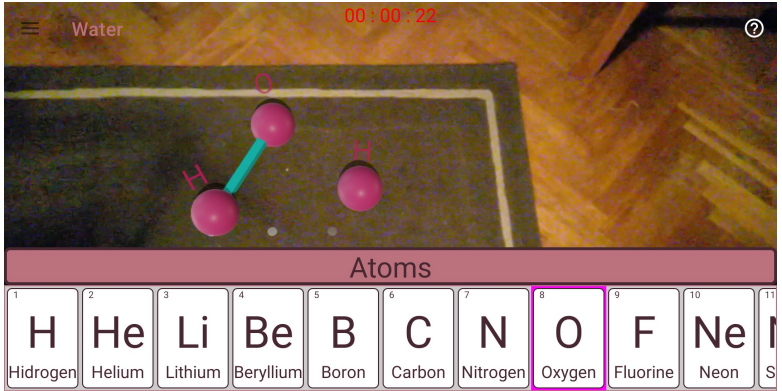

Figure 36. Bottom sheet menu in open state.

2. Action bar: This contains the icon through which you can access the menu ( $2 a$, top left), the name of the molecule $(2 b)$ and the help button $(2 d$, top right) through which you can open the help dialog again, which also appears at the beginning. Also, in this part of the screen $(2 c$, in the middle) there is the timer that measures the time spent to complete a structure. The timer starts after placing the first atom in the augmented space.

3. Floating action menu for bonds: With this menu a bond can be created between two selected atoms. By pressing the Bond button (bottom left) the menu with the types of chemical bonds is expanded, these being: single bond $(3 a)$, double bond $(3 b)$, triple bond $(3 c)$.

4. Floating action menu for actions: With the help of this menu the user can perform different actions. Everything can be reset $(4 a)$, which means deleting all atoms. One or more selected atoms can be deleted $(4 b)$. Screenshots can be taken $(4 c)$. The created structure of the molecule can be validated $(4 d)$.

After they are placed in the AR space, the atoms can be selected by tapping on the corresponding sphere. The model changes its color to indicate that it is selected. The highlighted atoms can be deleted with the Delete atom action (4b). If there are only two atom elements highlighted, they can be linked with a bond using the bonds floating action menu (3). Previously defined links can be deleted by tapping on the 3D cubes that represents them.

In the database, a molecule is described using a flexible model built for enabling the validation of the created structures without adding any interaction constraints (like atoms defining order). This model allows the user to start building the structure with any atom of his/her choosing and the order of placing the bonds is also irrelevant. The built structure can be compared to the one chosen at the beginning of the activity and validated by using the Validate action (4d) from group four.

The model is based on the fact that the visual representation of a molecule is highly customizable. The important 
aspects to consider are: (1) the type of atoms; (2) the number of atoms from each type; (3) the links defined between the atoms. The validation method is based on verifying if a molecule has the right number and right type of atoms, each having the appropriate bonds.

For motivating users, basic elements of gamification have been implemented into this educational application, in the form of a counter for measuring the time needed for constructing and validating a molecule structure. After validation, the player has the option to save his score and then later view the high scores.

\section{USABILITY TESTING}

Educational games should be developed taking into consideration the users' needs and feedback. A methodology for a future usability testing has been developed in order to measure the strengths and weaknesses of the application.

\section{Methodology}

The application should be evaluated from two different perspectives by people from the target demographic: teachers and middle school kids. A methodology has been design which will be implemented in the future for usability testing.

The test must include five phases for students. Because the purpose of the application is to support the memory and better understanding of molecular structures learned in chemistry class, the first part would be a normal class, held face to face with a chemistry teacher where they talk about some molecules detailing some of their physical properties and basic chemicals, about their molecular structure and about some interesting information. After the class, the students are required to do a quick test regarding the previously mentioned molecular structures.

In the second part, participants are handed the application already installed on a mobile phone other than their own. They are assigned two consecutive tasks:

1. Users are told to read the instructions carefully and interact with the application based on them. In this task they are required to start with a simple molecule with fewer atoms and bonds, such as sodium chloride or the hydrogen molecule. They are also asked to save their score and view the scores afterwards.

2. For the second task, participants have to repeat the same procedure, but this time before starting an oral explanation is held and questions were answered regarding the functionality.

In the third phase, participants are asked to complete a questionnaire about their experiences using the educational application developed. The questionnaire consists of two parts:
1. The demographic part referring to age, their chemical knowledge, their technical knowledge and related to augmented reality.

2. Application-specific information, recording feedback after using the application.

As a fourth phase students are required to retake the test to see if it affected their ability to memorize the certain structures.

As a last part, an unstructured discussion will take place, brainstorming with all participants at the same time, in which they can freely express their opinion on the application. Here they are meant to discuss the disadvantages and ideas related to improving the project.

Regarding teachers, their evaluation would consist of phases two, three and five of the methodology described before. They already have the necessary knowledge of chemistry so they cannot test memorization. Their opinion of the application's educational value would be quite important.

\section{CONCLUSION}

Augmented reality and virtual reality have huge potential in the field of education. These technologies combine different senses in the learning process and help to improve student involvement. The technology of choice had been augmented reality because of its many advantages. The fact that AR elements enrich reality makes it easier to abstract and capture complex ideas in chemistry. This way molecules can surround us everywhere visually too.

The research evaluated education assisted by information technology and the current state in the field. The technology of augmented reality and its possibilities in education were detailed. Following this study an educational mobile application was implemented, using the technology of augmented reality, in the field of chemistry. At the end a methodology for the usability testing of the application was proposed.

\section{FUTURE IMPROVEMENTS}

The developed application aims to provide an important tool for teachers and students in chemical education, namely easier practice and understanding of chemical structures.

One direction of development is the integration of a tangible augmented reality system. This would mean that the user interface would expand more in the real world and not just through the camera of the mobile device.

The current state of the application was assessed through a study. Based on this study, we can say that the serious game feature must be emphasized more strongly. The application could have an element of online community. That would mean the option to record and share the result with other users. This distribution functionality would also be great 
for sharing results with teachers. Challenges and levels can also be created for this purpose.

It would be advantageous to work on the better integration into formal education. For this purpose, a web or mobile platform should be developed for teachers through which they can add new molecular structures to, modify the details of existing molecules and view student activity. Through this platform it could create themes, new challenges for students.

\section{REFERENCES}

[1] A. H. Johnstone, "Why is science difficult to learn? Things are seldom what they seem," Journal of computer assisted learning, vol. 7, no. 2, pp. 75-83, 1991.

[2] P. Milgram and F. Kishino, "A taxonomy of mixed reality visual displays," IEICE TRANSACTIONS on Information and Systems, vol. 77, no. 12, pp. 13211329, 1994.

[3] C. Dede, "Immersive interfaces for engagement and learning," science, vol. 323, no. 5910, pp. 66-69, 2009.

[4] I. Radu, "Augmented reality in education: a metareview and cross-media analysis," Personal and Ubiquitous Computing, vol. 18, no. 6, pp. 1533-1543, 2014.

[5] K. Lee, "Augmented reality in education and training," TechTrends, vol. 56, no. 2, pp. 13-21, 2012.

[6] K.-H. Cheng and C.-C. Tsai, "Affordances of augmented reality in science learning: Suggestions for future research," Journal of science education and technology, vol. 22, no. 4, pp. 449-462, 2013.

[7] Y.-C. Chen, "A study of comparing the use of augmented reality and physical models in chemistry education," in Proceedings of the 2006 ACM international conference on Virtual reality continuum and its applications, 2006, pp. 369-372.

[8] X. A. Perkins Coie LLP. (2020) 2020 Augmented and Virtual Reality Survey Report. Industry Insights Into The Future of Immersive Technology.

[9] H. Kaufmann, "Construct3D: an augmented reality application for mathematics and geometry education," in Proceedings of the tenth ACM international conference on Multimedia, 2002, pp. 656-657.

[10] A. Abuhammad et al., "“MedChemVR": A Virtual Reality Game to Enhance Medicinal Chemistry Education," Multimodal Technologies and Interaction, vol. 5, no. 3, p. 10, 2021.

[11] M. Billinghurst and A. Duenser, "Augmented reality in the classroom," Computer, vol. 45 , no. 7 , pp. 56-63, 2012.

[12] O. A. Jones, M. Spichkova, and M. J. Spencer, "Chirality-2: Development of a multilevel mobile gaming app to support the teaching of introductory undergraduate-level organic chemistry," ed: ACS Publications, 2018.

[13] "EduChem VR." https://educhem-vr.com/ (accessed 2021).

[14] M. Núñez, R. Quirós, I. Núñez, J. B. Carda, E. Camahort, and J. Mauri, "Collaborative augmented reality for inorganic chemistry education," in WSEAS international conference. Proceedings. Mathematics and computers in science and engineering, 2008, vol. 5: WSEAS, pp. 271-277.

[15] F. M. Fung et al., "Applying a virtual reality platform in environmental chemistry education to conduct a field trip to an overseas site," ed: ACS Publications, 2019.

[16] "Atom Visualizer for ARCore." https://stem.signalgarden.com/ (accessed 2021).

[17] M. Fjeld and B. M. Voegtli, "Augmented chemistry: An interactive educational workbench," in Proceedings. International Symposium on Mixed and Augmented Reality, 2002: IEEE, pp. 259-321.

[18] P. Maier and G. Klinker, "Augmented chemical reactions: An augmented reality tool to support chemistry teaching," in 2013 2nd Experiment@ International Conference (exp. at'13), 2013: IEEE, pp. 164-165.

[19] "ARCore." https://developers.google.com/ar (accessed 2021).

[20] "Sceneform SDK." https://developers.google.com/sceneform/develop (accessed 2021). 